\title{
Succinate and Lactate Production from Euglena gracilis during Dark, Anaerobic Conditions
}

\author{
Yuko Tomita ${ }^{1}$, Kazumasa Yoshioka', Hiroko lijima ${ }^{1}$, Ayaka Nakashima², Osamu Iwata², \\ Kengo Suzuki², Tomohisa Hasunuma ${ }^{3}$, Akihiko Kondo ${ }^{3}$, Masami Yokota Hirai $^{4}$ and \\ Takashi Osanai ${ }^{1,4 *}$ \\ ${ }^{1}$ School of Agriculture, Meiji University, Kawasaki, Japan, ${ }^{2}$ euglena Co., Ltd, Tokyo, Japan, ${ }^{3}$ Graduate School of Science, \\ Technology and Innovation, Kobe University, Kobe, Japan, ${ }^{4}$ RIKEN Center for Sustainable Resource Science, Yokohama, \\ Japan
}

OPEN ACCESS

Edited by:

Weiwen Zhang,

Tianjin University, China

Reviewed by:

Jiangxin Wang,

Shenzhen University, China

Tanakarn Monshupanee,

Chulalongkorn University, Thailand

*Correspondence:

Takashi Osanai

tosanai@meiji.ac.jp

Specialty section:

This article was submitted to Microbiotechnology, Ecotoxicology and Bioremediation,

a section of the journal

Frontiers in Microbiology

Received: 25 October 2016 Accepted: 07 December 2016 Published: 21 December 2016

Citation

Tomita Y, Yoshioka K, lijima $H$, Nakashima A, Iwata O, Suzuki $K$, Hasunuma T, Kondo A, Hirai MY and

Osanai T (2016) Succinate and Lactate Production from Euglena gracilis during Dark, Anaerobic Conditions. Front. Microbiol. 7:2050.

doi: 10.3389/fmicb.2016.02050
Euglena gracilis is a eukaryotic, unicellular phytoflagellate that has been widely studied in basic science and applied science. Under dark, anaerobic conditions, the cells of E. gracilis produce a wax ester that can be converted into biofuel. Here, we demonstrate that under dark, anaerobic conditions, E. gracilis excretes organic acids, such as succinate and lactate, which are bulk chemicals used in the production of bioplastics. The levels of succinate were altered by changes in the medium and temperature during dark, anaerobic incubation. Succinate production was enhanced when cells were incubated in $\mathrm{CM}$ medium in the presence of $\mathrm{NaHCO}_{3}$. Excretion of lactate was minimal in the absence of external carbon sources, but lactate was produced in the presence of glucose during dark, anaerobic incubation. E. gracilis predominantly produced Llactate; however, the percentage of D-lactate increased to $28.4 \%$ in CM medium at $30^{\circ} \mathrm{C}$. Finally, we used a commercial strain of $E$. gracilis for succinate production and found that nitrogen-starved cells, incubated under dark, anaerobic conditions, produced 869.6 mg/L succinate over a 3-day incubation period, which was 70-fold higher than the amount produced by nitrogen-replete cells. This is the first study to demonstrate organic acid excretion by $E$. gracilis cells and to reveal novel aspects of primary carbon metabolism in this organism.

Keywords: biorefinery, Euglena, lactate, microalgae, succinate

\section{INTRODUCTION}

Euglena gracilis is a eukaryotic, unicellular phytoflagellate in the genus Euglena, characterized by dynamic and flexible cell structure, and commercially cultivated as a nutritional food. E. gracilis has also been studied as a biocatalyst for the production of bioenergy. E. gracilis cells fix carbon dioxide $\left(\mathrm{CO}_{2}\right)$ and produce the polysaccharide paramylon, a $\beta$-1,3-glucan (Calvayrac et al., 1981). Paramylon stored under aerobic conditions is degraded during anaerobic cultivation to produce a wax ester consisting of saturated fatty acids and alcohol chains (Inui et al., 1984). Wax esters act as electron sinks from glycolytic ATP and pyruvate oxidation under anaerobic conditions (Hoffmeister et al., 2005). Under aerobic conditions, fatty acid biosynthesis starts with pyruvate and acetyl-CoA in the mitochondria (Inui et al., 1984); therefore, pyruvate and acetyl-CoA metabolism varies widely depending on the growth conditions. Adding acetate or ethanol into the medium 
increases the activities of acetate kinase, malate synthase and isocitrate lyase, suggesting that acetate is assimilated and metabolized through the glyoxylate pathway (Woodward and Merrett, 1975; Nakazawa et al., 2005). External ethanol is metabolized by mitochondrial alcohol dehydrogenase, which can assimilate other alcohols such as 1-butanol and 1-heptanol (Ono et al., 1995). Thus, the metabolism of pyruvate, acetylCoA, and organic acids in the tricarboxylic acid (TCA) cycle is dramatically changed by the growth conditions. The effect of external metabolites on primary carbon metabolism inside the cells is determined by several groups. However, the excretion of metabolites from carbon metabolism has only been detailed in the wax ester.

Succinate production has been intensively evaluated in heterotrophic bacteria because of their importance in biorefinery. Succinate is used as a building block for various compounds including the widely used plastic polybutylene succinate. Succinate is produced petrochemically, but bio-succinate production is preferable for reducing the environmental burden, as proposed by the United States Department of Energy (Werpy and Petersen, 2004). Succinate production using heterotrophic bacteria requires external carbon sources such as glucose; however, direct conversion of $\mathrm{CO}_{2}$ is preferable to avoid competition with foods. Recently, it was found that several cyanobacteria excrete organic acids under dark, anaerobic conditions (McNeely et al., 2014; Osanai et al., 2015; Iijima et al., 2016; Ueda et al., 2016). Cyanobacterial cells accumulate glycogen via fixation of $\mathrm{CO}_{2}$ under photoautotrophic conditions and glycogen is degraded under dark, anaerobic conditions. These compounds are converted into organic acids, such as lactate, acetate, and succinate, which are excreted under dark, anaerobic conditions (McNeely et al., 2014; Osanai et al., 2015; Hasunuma et al., 2016; Iijima et al., 2016; Ueda et al., 2016). Recently, succinate levels have reached $430 \mathrm{mg} / \mathrm{L}$ by metabolic engineering of cyanobacteria (Lan and Wei, 2016). This process is known as autofermentation and the organic acids are produced from $\mathrm{CO}_{2}$ by cyanobacteria as biocatalysts.

In this study, we found that the cells of E. gracilis excrete succinate under dark, anaerobic conditions. Lactate was also excreted from the cells following the addition of glucose, revealing valuable chemical production by E. gracilis.

B

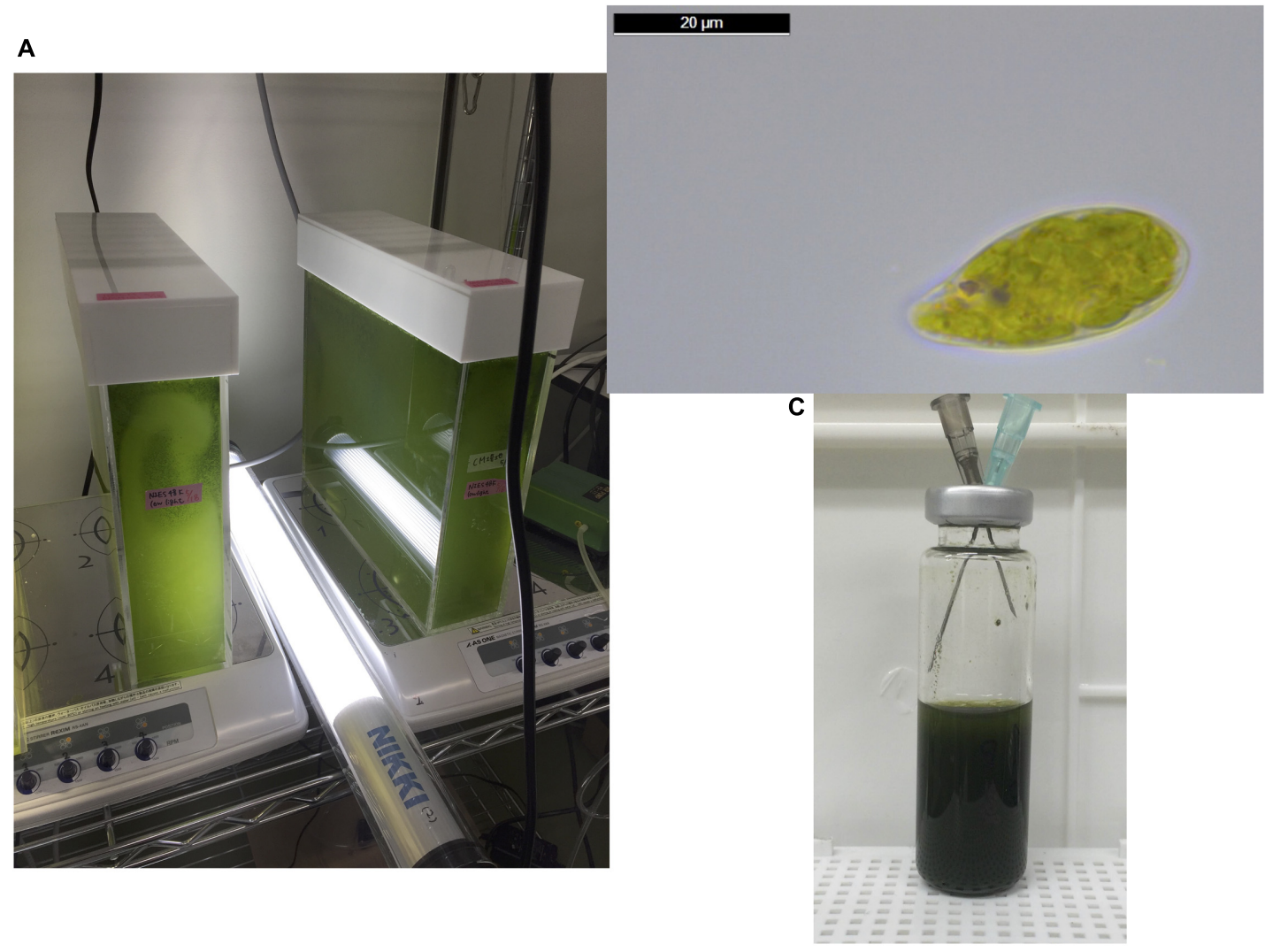

FIGURE 1 | (A) The cells of Euglena gracilis were cultured at approximately $25^{\circ} \mathrm{C}$ in a $5 \mathrm{~L}$ acrylic tank placed on a magnetic stirrer under an LED light with an intensity of $60 \mu \mathrm{mol}$ photons $\mathrm{m}^{-2} \mathrm{~s}^{-1}$. (B) A cell of $E$. gracilis grown under photoautotrophic conditions, observed using a Leica DM500 microscope (Leica Microsystems, Tokyo, Japan). The image was analyzed using the Leica Application Suite software, version 4.6. (C) Anaerobic incubation for excretion of organic acid. Cells were concentrated in $10 \mathrm{~mL} C M$ medium or HEPES buffer at $A_{730}=20$ in a $20 \mathrm{~mL} \mathrm{GC}$ vial. The vial was sealed with a butyl rubber cap and nitrogen gas was introduced using syringes for $1 \mathrm{~h}$. After removing the syringes, the vial was wrapped with aluminum foil and incubated for 3 days with shaking at 25 or $30^{\circ} \mathrm{C}$. 


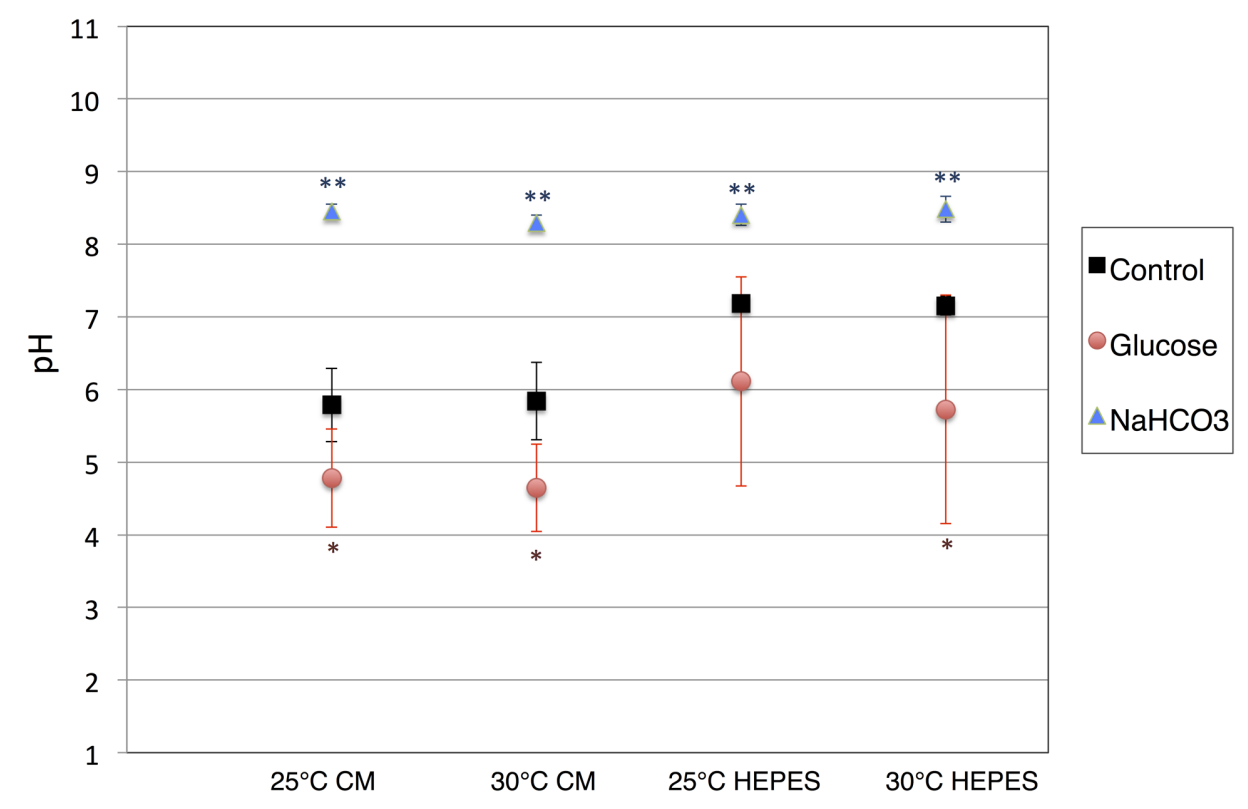

FIGURE 2 | pH of the supernatants after dark, anaerobic incubation. The cells of $E$. gracilis were incubated under dark, anaerobic conditions for 3 days in CM medium or HEPES buffer with/without $100 \mathrm{mM}$ glucose and $100 \mathrm{mM} \mathrm{NaHCO}$. After centrifugation to remove the cells, the pH of the supernatants was quantified. Data represent the mean \pm SD from biologically independent samples $(n=5-7)$. Asterisks indicate statistically significant differences in pH from supernatants collected from cells grown with and without external carbon sources (Student's $t$-test; ${ }^{*} P<0.05,{ }^{* *} P<0.005$ ).

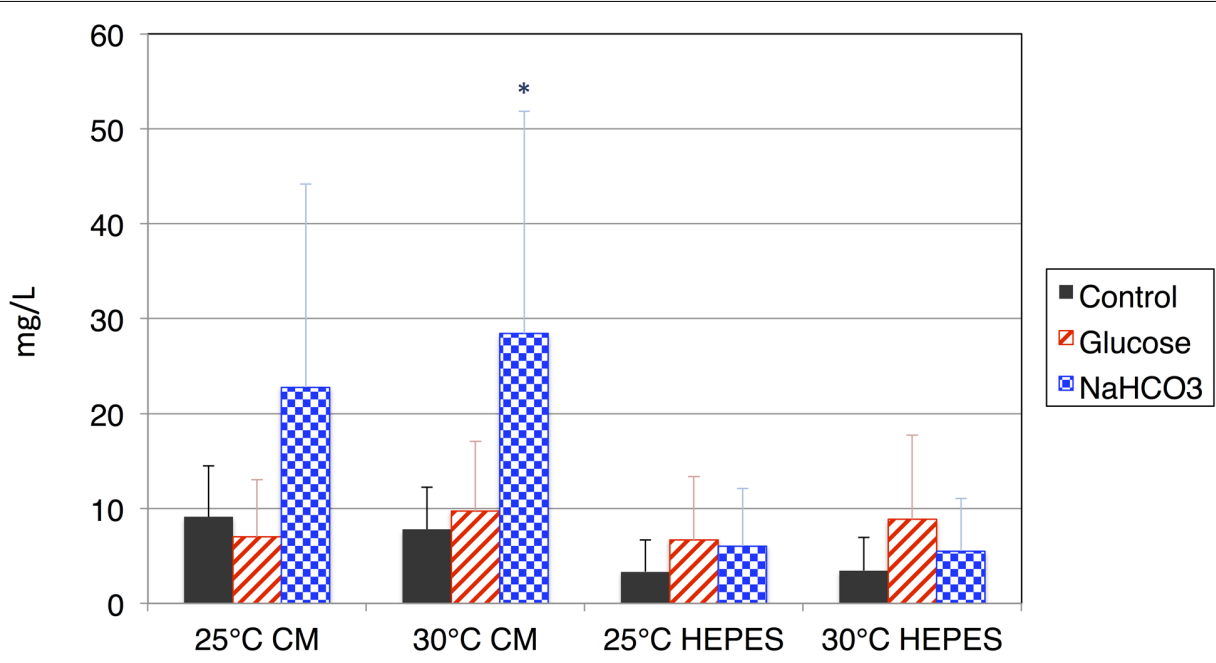

FIGURE 3 | Levels of succinate produced by cells of $\boldsymbol{E}$. gracilis incubated under dark, anaerobic conditions. The cells of $E$. gracilis were incubated under

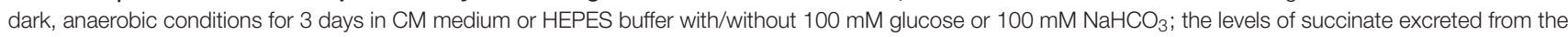
cells were quantified using HPLC. Data represent the mean \pm SD from biologically independent samples $(n=6-10)$. Asterisk indicates statistically significant differences in the levels of succinate produced with and without external carbon sources (Student's $t$-test; $\left.{ }^{*} P<0.05\right)$.

\section{MATERIALS AND METHODS}

\section{Euglena Strains and Culture Conditions}

Two strains of E. gracilis, NIES-48 from the National Institute for Environmental Studies, Japan and commercial strain from euglena, Co., Ltd, (Tokyo, Japan) (Yamada et al., 2016) were used in this study. E. gracilis cells were grown in modified CM medium (adjusted to pH 3.5) (Cramer and Myers, 1952). For preculture, cells were grown in liquid medium bubbled with $1 \%(\mathrm{v} / \mathrm{v}) \mathrm{CO}_{2}$ in air and incubated in a plant growth chamber (TOMY, Tokyo, Japan) at $25^{\circ} \mathrm{C}$ under $12 \mathrm{~h}$ light $/ 12 \mathrm{~h}$ dark conditions with white light at $\sim 40 \mu \mathrm{mol}$ photons $\mathrm{m}^{-2} \mathrm{~s}^{-1}$. After preculture, the cells were inoculated into $5 \mathrm{~L}$ of modified $\mathrm{CM}$ medium containing $10 \mathrm{mM} \mathrm{NaHCO}_{3}$ in an acrylic tank at room temperature (approximately $25^{\circ} \mathrm{C}$ ) and cultivated for approximately 15 days. Cell cultures were mixed with a magnetic stirrer and exposed 

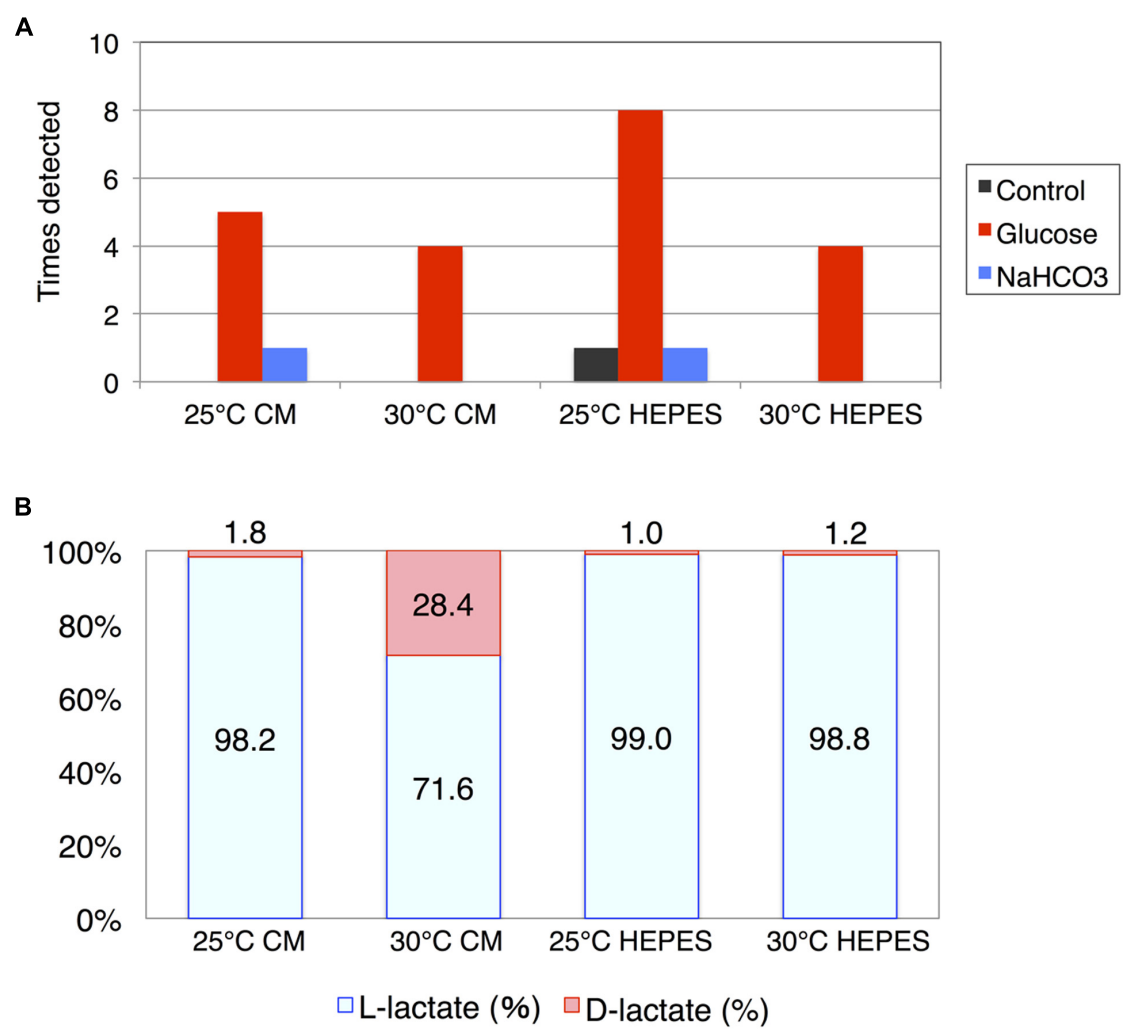

FIGURE 4 | Lactate production by cells of $\boldsymbol{E}$. gracilis incubated under dark, anaerobic conditions. (A) The number of times lactate was detected using HPLC. The cells of E. gracilis were incubated under dark, anaerobic conditions for 3 days in CM medium or HEPES buffer with/without $100 \mathrm{mM}$ glucose and $100 \mathrm{mM} \mathrm{NaHCO}_{3}$. Experiments were repeated 10 times and the number of times lactate was detected at more than $10 \mathrm{mg} / \mathrm{L}$ was counted and visualized. (B) Percentage of L-lactate and D-lactate. Lactates from E. gracilis cells incubated under dark, anaerobic conditions for 3 days in CM medium or HEPES buffer containing $100 \mathrm{mM}$ glucose were analyzed. Two replicates of each sample, containing the highest level of lactate, were used to determine chirality.

to white light using LED at $60 \mu \mathrm{mol}$ photons $\mathrm{m}^{-2} \mathrm{~s}^{-1}$. For nitrogen depletion, $\left(\mathrm{NH}_{4}\right)_{2} \mathrm{HPO}_{4}$ was replaced with $\mathrm{KH}_{2} \mathrm{PO}_{4}$. Cell densities were measured at $A_{730}$ using a Shimadzu UV-2400 spectrophotometer (Shimadzu, Kyoto, Japan).

\section{Dark, Anaerobic Incubation}

Dark, anaerobic incubation was performed as described previously (Osanai et al., 2015) with modifications. Cells were concentrated in $10 \mathrm{~mL}$ of modified CM medium or HEPES buffer (20 mM HEPES-KOH, pH 7.8) at $A_{730}=20$ in a GC vial; equal volumes of cultures were concentrated when using the commercial strain. The vials were sealed with a butyl rubber cap and nitrogen gas was introduced using syringes for $1 \mathrm{~h}$. Anaerobic conditions were maintained by removing the syringes and wrapping the vials with aluminum foil, followed by shaking at 25 or $30^{\circ} \mathrm{C}$ for 3 days. After cultivation, the cell cultures were centrifuged at $5800 \times g$ for $2 \mathrm{~min}$, the supernatant was filtered, and $1 \mathrm{~mL}$ of supernatant was freeze-dried for 1 day. The $\mathrm{pH}$ of the supernatants was measured using a LAQUAact $\mathrm{pH}$ meter (Horiba, Kyoto, Japan). Organic acids in the dried sample were analyzed by high performance liquid chromatography (HPLC). For the experiment using the commercial strain, cell cultures were concentrated from the volumes of 200 or $800 \mathrm{~mL}$ to those of $10 \mathrm{~mL}$ in HEPES buffer and similarly incubated under dark, anaerobic conditions; excreted organic acids were then measured using HPLC.

\section{Measurement of Excreted Organic Acids Using HPLC}

Organic acids were analyzed as described previously (Osanai et al., 2015). Freeze-dried supernatants were resolved in $100 \mu \mathrm{L}$ of filtered $3 \mathrm{mM}$ perchloric acid. The resolved samples were analyzed by HPLC using an LC-2000Plus System (JASCO, Tokyo, Japan) with a photodiode array detector and two RSpak KC811 columns (Showa Denko, Tokyo, Japan). Organic acids were quantified using $0.2 \mathrm{mM}$ bromothymol blue in $15 \mathrm{mM}$ sodium phosphate buffer; peaks were detected at $445 \mathrm{~nm}$. The temperature of the column was $60^{\circ} \mathrm{C}$, and flow rates of $3 \mathrm{mM}$ perchloric acid and $0.2 \mathrm{mM}$ bromothymol blue solutions were 0.7 and $1.2 \mathrm{~mL} / \mathrm{min}$, respectively.

\section{Determination of Proportion of Lactate Isomers}

Lactates were quantified using the F-Kit D-lactate/L-lactate (JK International, Tokyo, Japan) according to the manufacturer's instruction. Fifty microliters of the supernatant was mixed with 
$610 \mu \mathrm{L}$ glycylglycine buffer ( $\mathrm{pH}$ 10.0) containing $38.4 \mathrm{mM}$ glutamate, $22.3 \mathrm{mM} \mathrm{NAD}, 15.7 \mathrm{U}$ glutamate-pyruvate transaminase solution and sterilized distilled water for a total volume of $1 \mathrm{~mL}$. Absorbance was measured at $340 \mathrm{~nm}$ before and after the addition of $20 \mu \mathrm{L}$ of L-lactate or D-lactate dehydrogenase for $5 \mathrm{~min}$ (final concentrations of L-/D- lactate dehydrogenases were $108 \mathrm{U})$.

\section{RESULTS}

\section{Cultivation of $\boldsymbol{E}$. gracilis Cells and Succinate Production}

The cells of E. gracilis NIES-48 were grown in a $5 \mathrm{~L}$ acrylic tank placed on a magnetic stirrer (Figure 1A). Cells were grown at room temperature (approximately $25^{\circ} \mathrm{C}$ ) under white light from an LED at $60 \mu \mathrm{mol}$ photons $\mathrm{m}^{-2} \mathrm{~s}^{-1}$. Organic acid excretion from E. gracilis cells was assessed as described previously for cyanobacteria (Osanai et al., 2015). Cells grown under photoautotrophic conditions (Figure 1B) were concentrated in CM medium ( $\mathrm{pH} 3.5$ ) or HEPES buffer ( $\mathrm{pH} 7.8$ ) in a GC vial (Figure 1C), and incubated under dark, anaerobic conditions for 3 days at 25 and $30^{\circ} \mathrm{C}$. Additionally, $100 \mathrm{mM}$ glucose or $100 \mathrm{mM} \mathrm{NaHCO}$ was added as a source of carbon during the incubation. In the absence of external carbon sources, the $\mathrm{pH}$ of the supernatant, after a 3-day incubation in CM medium and HEPES buffer, was 5.8 and 7.2, respectively, regardless of the temperature (Figure 2). The addition of glucose reduced the $\mathrm{pH}$ by $0.9-1.5$ in both CM medium and HEPES buffer (Figure 2). The addition of $\mathrm{NaHCO}_{3}$ increased the $\mathrm{pH}$ to 8.3-8.5 under all four conditions (Figure 2).

Organic acids excreted from the cells were quantified using HPLC, and succinate was detected in the supernatant after dark, anaerobic incubation. Overall, the levels of succinate excreted from the cells incubated in CM medium were higher than those from cells incubated in HEPES buffer at both 25 and $30^{\circ} \mathrm{C}$ (Figure 3). The addition of glucose increased the levels of succinate in HEPES buffer, but decreased those in the CM medium (Figure 3). The addition of $\mathrm{NaHCO}_{3}$ increased the levels of succinate under all four conditions, regardless of the medium and temperature (Figure 3). The maximum concentration of succinate obtained from cells incubated in the CM medium containing $\mathrm{NaHCO}_{3}$ at $30^{\circ} \mathrm{C}$ was $28.4 \mathrm{mg} / \mathrm{L}$.

\section{Lactate Production}

In addition to succinate, HPLC analysis revealed that lactate was produced by the cells of E. gracilis during dark, anaerobic incubation. In contrast to succinate, lactate production showed poor reproducibility. The experiment was repeated 10 times and the number of experiments in which lactate was produced at a concentration greater than $10 \mathrm{mg} / \mathrm{L}$ was counted. Lactate was minimally detected when the cells were incubated under dark, anaerobic conditions without external sources of carbon (Figure 4A). However, lactate was produced in the presence of glucose as an external carbon source. Lactate production was observed eight times when cells were incubated in HEPES buffer at $25^{\circ} \mathrm{C}$ (Figure $4 \mathbf{A}$ ). $\mathrm{NaHCO}_{3}$ had a weaker effect on lactate

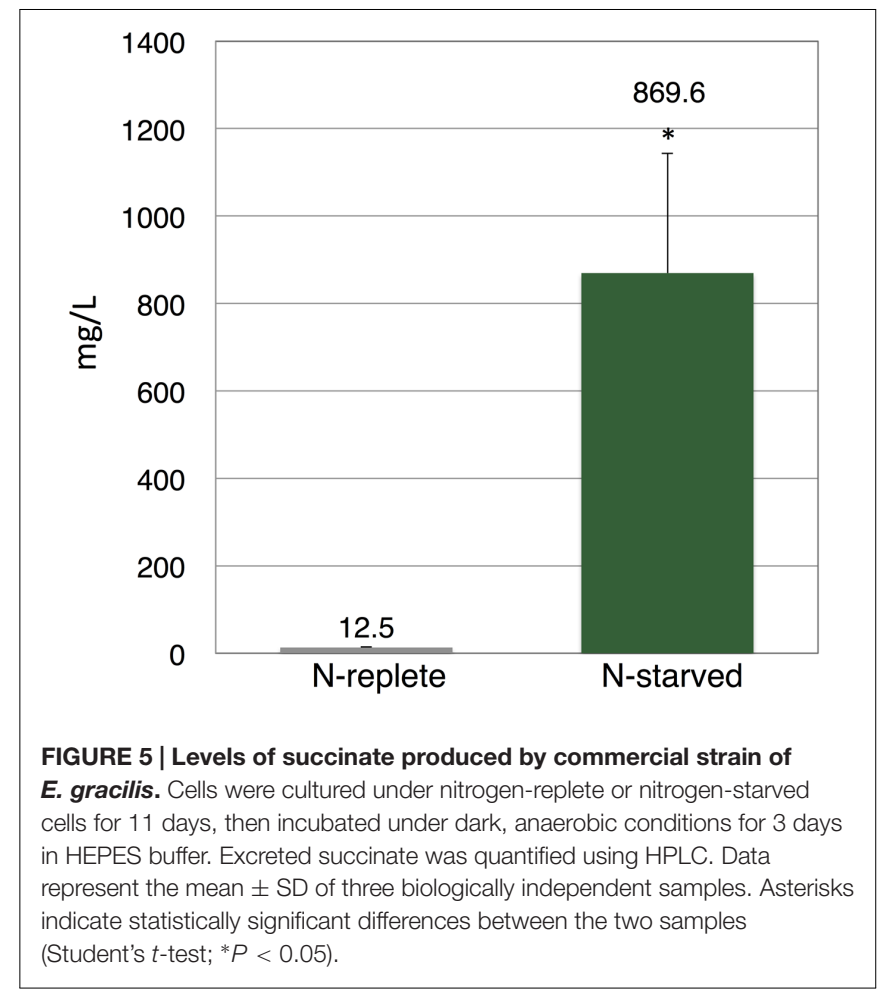

production compared with that of glucose (Figure 4A). The highest level of lactate was, produced by cells incubated in HEPES buffer containing $100 \mathrm{mM}$ glucose at $25^{\circ} \mathrm{C}$, was $1.4 \mathrm{~g} / \mathrm{L}$.

Because lactate is chiral, we enzymatically determined the ratio of L-/D-lactate produced from E. gracilis cells incubated in the presence of glucose; we used two replicates of each sample containing the highest levels of lactate. While both L-lactate and D-lactate enantiomers were detected; L-lactate predominated (Figure 4B). However, when the cells were incubated in $\mathrm{CM}$ medium at $30^{\circ} \mathrm{C}$ under dark, anaerobic conditions, the percentage of D-lactate was increased to $28.4 \%$ (Figure 4B).

\section{Succinate Production Using Commercial Strain of $E$. gracilis}

Finally, to evaluate the applicability of our method, we used the commercial strain of E. gracilis, which is derived from NIES-48 and has been commercially cultivated by the euglena, Co., Ltd, for 10 years (Yamada et al., 2016). To precisely control the growth conditions, the cells were cultivated in a plant growth chamber at $25^{\circ} \mathrm{C}$ under white light exposure (12 h light/12 h dark). The production of wax ester is enhanced by nitrogen starvation before dark, anaerobic cultivation (Arashida and Mitra, 2011). For nitrogen starvation, cells were cultivated for 11 days in nitrogen-depleted medium. Then, nitrogen-replete and nitrogenstarved cells, each in a volume of $800 \mathrm{~mL}$ culture medium, were concentrated and incubated under dark, anaerobic conditions. To reduce cost, all anaerobic incubation was performed in HEPES buffer without external carbon sources. The succinate level from nitrogen-replete cells was $12.5 \mathrm{mg} / \mathrm{L}$ (Figure 5). Succinate levels from nitrogen-starved cells reached $869.6 \mathrm{mg} / \mathrm{L}$, 


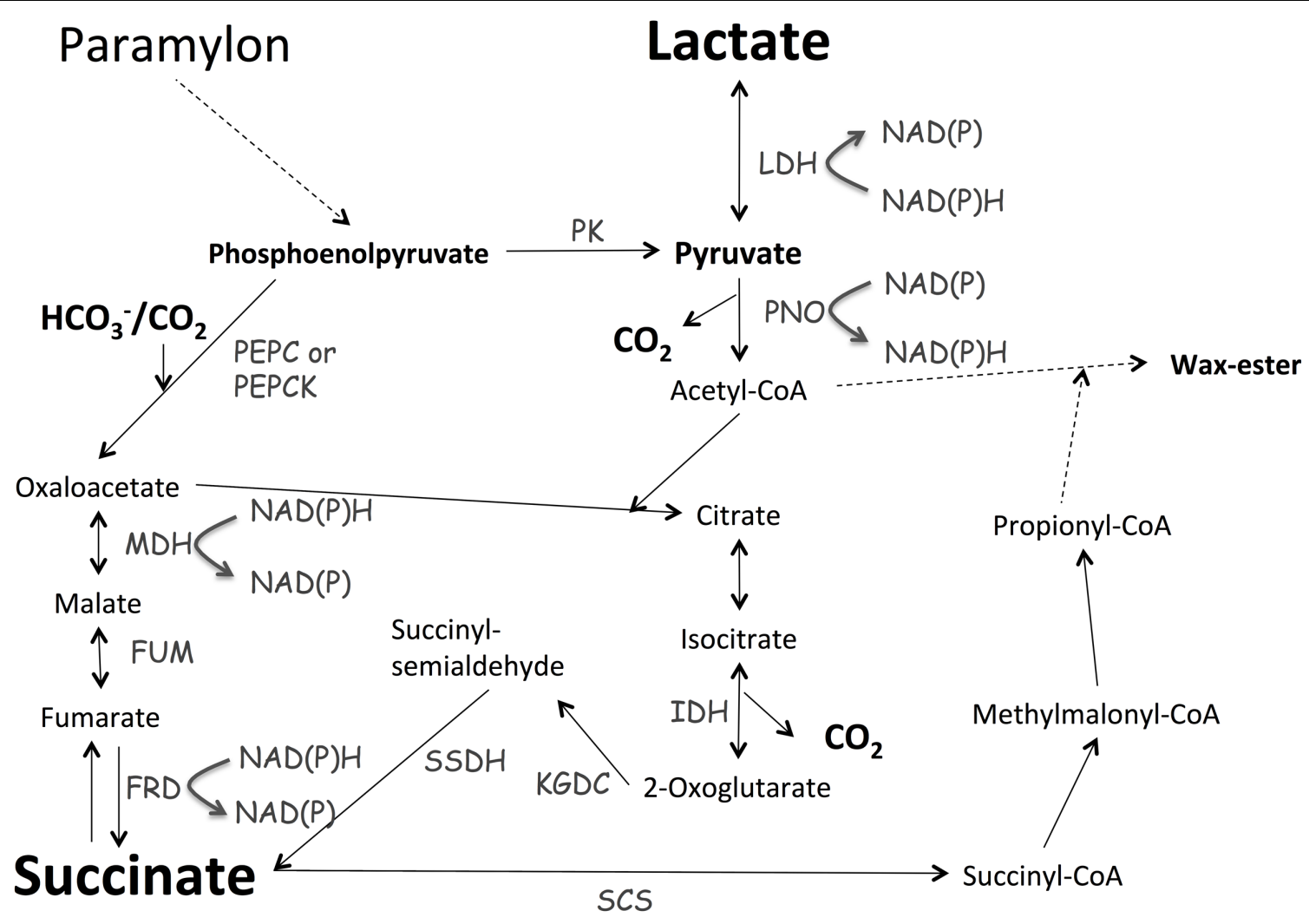

FIGURE 6 | Metabolic map describing lactate and succinate production. Metabolic map was generated based on the map described by Padermshoke et al. (2016). MDH, malate dehydrogenase; FRD, fumarate reductase; FUM, fumarase; IDH, isocitrate dehydrogenase; KGDC, $\alpha$-ketoglutarate dehydrogenase; LDH, lactate dehydrogenase; PEPC, phosphoenolpyruvate carboxylase; PEPCK, phosphoenolpyruvate carboxykinase; PK, pyruvate kinase; PNO, pyruvate:NADP+ oxidoreductase; SSDH, succinyl-semialdehyde dehydrogenase.

which was 70 -fold higher than that from nitrogen-replete cells (Figure 5). Addition of external carbon sources or potassium, which enhanced succinate production in Synechocystis sp. PCC 6803 (Ueda et al., 2016), did not increase succinate levels from nitrogen-starved cells (Supplementary Figure S1).

\section{DISCUSSION}

In this study, we found that E. gracilis, transferred from light, aerobic conditions to dark, anaerobic conditions, excreted succinate and lactate during incubation. These metabolites are typical fermentative products excreted under dark, anaerobic conditions to oxidize $\mathrm{NAD}(\mathrm{P}) \mathrm{H}$ for substrate-level phosphorylation. Controlling growth conditions is important for biochemical production using E. gracilis (Grimm et al., 2015). The levels of $\alpha$-tocopherol in cells grown under photoautotrophic conditions are 1.6-fold higher than those in cells grown under heterotrophic conditions (Grimm et al., 2015). The levels of paramylon in cells grown heterotrophically are 12-fold higher than those in cells grown photoautotrophically (Grimm et al., 2015). Adding glucose and ethanol into the medium increases paramylon production in E. gracilis (Rodríguez-Zavala et al., 2010). Here, we showed that changing the medium and temperature under dark, anaerobic conditions altered the levels of succinate from E. gracilis (Figure 3). Lactate production was dependent on supplementation with external glucose, and chirality was altered temperature and type of medium used (Figure 4B). The poor reproducibility of lactate production indicates that lactate excretion and biosynthesis are controlled by several factors in addition to temperature and type of medium (Figure 4A). Because the genomic sequence of $E$. gracilis has not been determined, the number of lactate dehydrogenases is unknown. The activity of L-lactate and D-lactate dehydrogenase has been shown in the mitochondria and cytosol of E. gracilis (Jasso-Chávez et al., 2001, 2005). Thus, the production of both isomers using E. gracilis is rational; nevertheless the physiological relevance requires further examination. In the case of Lactobacillus coryniformis, L-lactate dehydrogenase is more stable under heat treatment than Dlactate dehydrogenase is; this stability results in increased levels of L-lactate at higher fermentation temperature (Gu et al., 2014). Thus, thermostabilities of lactate dehydrogenases determine the ratio of L-lactate/D-lactate. Analysis of lactate dehydrogenases in E. gracilis shows that D-lactate dehydrogenase is more tolerant to high temperature than L-lactate dehydrogenase is (Jasso-Chávez et al., 2001), which is consistent with our result (Figure 4B). The production of lactate and succinate requires 
oxidation of $\mathrm{NAD}(\mathrm{P}) \mathrm{H}$ for substrate-level phosphorylation (Figure 6). As shown in the metabolic map, succinate production requires additional carbon fixation by $\mathrm{PEPC/PEPCK}$, while lactate production does not (Figure 6). This may be the reason for increased lactate production in the presence of glucose (Figure 4A).

Recently, two independent groups analyzed the transcriptome of E. gracilis (O’Neill et al., 2015; Yoshida et al., 2016) and found that E. gracilis contains approximately 50,000 unique transcripts; 800 and 1280 transcripts were upregulated and downregulated by a 24-h anaerobic incubation, respectively (Yoshida et al., 2016). The expression of genes involved in photosynthesis, nucleotide metabolism, TCA cycle, and oxidative phosphorylation was downregulated under anaerobic conditions (Yoshida et al., 2016). Yoshida et al. (2016) proposed E. gracilis uses fumarate, rather than $\mathrm{O}_{2}$, as an electron acceptor under anaerobic conditions; this agrees with our results showing that E. gracilis produced succinate during dark, anaerobic incubation (Figure 3). Metabolomic analysis, conducted using the same strain of E. gracilis, shows that compared with the levels of metabolites in cells grown under light, aerobic conditions, the levels of pyruvate decreased to $4 \%$ in cells grown under dark, anaerobic conditions (Matsuda et al., 2011). The authors suggest that the decrease in pyruvate was related to the biosynthesis of wax ester, which is produced from acetyl-CoA (Matsuda et al., 2011); however, we suggest that the decreased pyruvate is also related to the production of succinate and lactate under dark, anaerobic conditions (Figures 3 and 4). Although the biosynthetic pathway of succinate is currently unclear, in the unicellular cyanobacterium Synechocystis sp. PCC 6803, succinate biosynthesis under dark, anaerobic conditions begins with the conversion of phosphoenolpyruvate to oxaloacetate via phosphoenolpyruvate carboxylase (PEPC) and through the reductive TCA cycle (Hasunuma et al., 2016). In E. gracilis, phosphoenolpyruvate carboxykinase (PEPCK) assimilates $\mathrm{HCO}_{3}{ }^{-}$under dark, anaerobic conditions (Padermshoke et al., 2016). Combined with the results of transcriptomic and metabolomic analyses (Yoshida et al., 2016), our results suggest that E. gracilis produces succinate via the reductive TCA cycle under dark, anaerobic conditions (Figure 6); to confirm this notion, metabolic flux analysis must be performed. Under dark, anaerobic conditions, dihydroxyacetone phosphate accumulates in E. gracilis cells (Matsuda et al., 2011), and Synechocystis sp. PCC 6803 (Osanai et al., 2015). Conversely, the effect of potassium on succinate production in Synechocystis sp. PCC 6803 differs from that in E. gracilis (Supplementary Figure S1; Ueda et al., 2016). Thus, comparing the results of metabolomic

\section{REFERENCES}

Arashida, R., and Mitra, S. (2011). Method for production of euglena containing wax ester at high content, and method for production of wax ester. US 20130115666 A1.

Briand, J., Calvayrac, R., Laval-Martin, D., and Farineau, J. (1981). Evolution of carboxylating enzymes involved in paramylon synthesis (phosphoenolpyruvate carboxylase and carboxykinase) in heterotrophically grown Euglena gracilis. Planta 151, 168-175. doi: 10.1007/BF00387819 analyses will help to elucidate the differences in primary carbon metabolism among photosynthetic organisms grown under anaerobic conditions. Carboxylation of phosphoenolpyruvate by PEPC or PEPCK is a rate-limiting step during succinate production in heterotrophic bacteria (Millard et al., 1996; Lee et al., 2006; Tan et al., 2013). $\mathrm{HCO}_{3}{ }^{-}$is a substrate of PEPCK/PEPC (Figure 6); therefore, the addition of $\mathrm{NaHCO}_{3}$ promoted succinate production in E. gracilis. PEPC activity in E. gracilis increases at the end of the exponential phase, possibly because an increase in gluconeogenesis is needed to produce paramylon (Briand et al., 1981). Therefore, the status of the cells before dark, anaerobic incubation is also important for succinate production, as was demonstrated using the commercial strain in this study (Figure 5). Supplementation with external sources of carbon did not increase succinate production in the nitrogenstarved cells (Supplementary Figure S1), indicating that carbon sources are not rate-limiting for nitrogen-starved cells because of high accumulation of paramylon induced by nitrogen starvation. Our results demonstrate that E. gracilis can be used to produce various chemicals as biocatalysts for microbial cell factories.

\section{AUTHOR CONTRIBUTIONS}

YT, KY, HI, and TO performed the experiments and analyzed the data, AN, OI, KS, TH, AK, and MH, designed the study, and TO wrote the manuscript.

\section{ACKNOWLEDGMENTS}

This work was supported by the Ministry of Education, Culture, Sports, Science, and Technology, Japan; by a grant to TO from ALCA (Project name "Production of cyanobacterial succinate by the genetic engineering of transcriptional regulators and circadian clocks") from the Japan Science and Technology Agency and by JSPS KAKENHI Grant-in-Aid for Scientific Research on Innovative Areas Grant Number 16H06559. This study was also funded by euglena Co., Ltd. Funding sources did not affect the interpretation of the results in this study.

\section{SUPPLEMENTARY MATERIAL}

The Supplementary Material for this article can be found online at: http://journal.frontiersin.org/article/10.3389/fmicb. 2016.02050/full\#supplementary-material

Calvayrac, R., Laval-Martin, D., Briand, J., and Farineau, J. (1981). Paramylon synthesis by Euglena gracilis photoheterotrophically grown under low $\mathrm{O} 2$ pressure: description of a mitochloroplast complex. Planta 153, 6-13. doi: 10. 1007/BF00385311

Cramer, M., and Myers, J. (1952). Growth and photosynthetic characteristics of Euglena gracilis. Arch. Microbiol. 17, 384-402. doi: 10.1007/BF00410835

Grimm, P., Risse, J. M., Cholewa, D., Müller, J. M., Beshay, U., Friehs, K., et al. (2015). Applicability of Euglena gracilis for biorefineries demonstrated by the production of $\alpha$-tocopherol and paramylon followed by 
anaerobic digestion. J. Biotechnol. 215, 72-79. doi: 10.1016/j.jbiotec.2015. 04.004

Gu, S.-A., Jun, C., Joo, J. C., Kim, S., Lee, S. H., and Kim, Y. H. (2014). Higher thermostability of l-lactate dehydrogenases is a key factor in decreasing the optical purity of d-lactic acid produced from Lactobacillus coryniformis. Enzyme Microb. Technol. 5, 29-35. doi: 10.1016/j.enzmictec.2014.02.008

Hasunuma, T., Matsuda, M., and Kondo, A. (2016). Improved sugar-free succinate production by Synechocystis sp. PCC 6803 following identification of the limiting steps in glycogen catabolism. Metab. Eng. Commun. 3, 130-141.

Hoffmeister, M., Piotrowski, M., Nowitzki, U., and Martin, W. (2005). Mitochondrial trans-2-enoyl-CoA reductase of wax ester fermentation from Euglena gracilis defines a new family of enzymes involved in lipid synthesis. J. Biol. Chem. 280, 4329-4338. doi: 10.1074/jbc.M411010200

Iijima, H., Shirai, T., Okamoto, M., Pinto, F., Tamagnini, P., Hasunuma, T., et al. (2016). Metabolomics-based analysis revealing the alteration of primary carbon metabolism by the genetic manipulation of a hydrogenase $\mathrm{HoxH}$ in Synechocystis sp. PCC 6803. Algal Res. 18, 305-313.

Inui, H., Miyatake, K., Nakano, Y., and Kitaoka, S. (1984). Fatty acid synthesis in mitochondria of Euglena gracilis. Eur. J. Biochem. 142, 121-126. doi: 10.1111/j. 1432-1033.1984.tb08258.x

Jasso-Chávez, R., García-Cano, I., Marín-Hernández, A., Mendoza-Cózatl, D., Rendón, J. L., and Moreno-Sánchez, R. (2005). The bacterial-like lactate shuttle components from heterotrophic Euglena gracilis. Biochim. Biophys. Acta 1709, 181-190. doi: 10.1016/j.bbabio.2005.07.007

Jasso-Chávez, R., Torres-Márquez, M. E., and Moreno-Sánchez, R. (2001). The membrane-bound 1- and d-lactate dehydrogenase activities in mitochondria from Euglena gracilis. Arch. Biochem. Biophys. 390, 295-303. doi: 10.1006/abbi. 2001.2353

Lan, E. I., and Wei, C. T. (2016). Metabolic engineering of cyanobacteria for the photosynthetic production of succinate. Metab. Eng. 38, 483-493. doi: 10.1016/ j.ymben.2016.10.014

Lee, S. J., Song, H., and Lee, S. Y. (2006). Genome-based metabolic engineering of Mannheimia succiniciproducens for succinic acid production. Appl. Environ. Microbiol. 72, 1939-1948. doi: 10.1128/AEM.72.3.1939-1948.2006

Matsuda, M., Hayashi, M., and Kondo, A. (2011). Comparative profiling analysis of central metabolites in Euglena gracilis under various cultivation conditions. Biosci. Biotechnol. Biochem. 75, 2253-2256. doi: 10.1271/bbb.110482

McNeely, K., Kumaraswamy, G. K., Guerra, T., Bennette, N., Ananyev, G., and Dismukes, G. C. (2014). Metabolic switching of central carbon metabolism in response to nitrate: application to autofermentative hydrogen production in cyanobacteria. J. Biotechnol. 18, 83-91. doi: 10.1016/j.jbiotec.2014.04.004

Millard, C. S., Chao, Y. P., Liao, J. C., and Donnelly, M. I. (1996). Enhanced production of succinic acid by overexpression of phosphoenolpyruvate carboxylase in Escherichia coli. Appl. Environ. Microbiol. 62, 1808-1810.

Nakazawa, M., Minami, T., Teramura, K., Kumamoto, S., Hanato, S., Takenaka, S., et al. (2005). Molecular characterization of a bifunctional glyoxylate cycle enzyme, malate synthase/isocitrate lyase, in Euglena gracilis. Comp. Biochem. Physiol. B Biochem. Mol. Biol. 141, 445-452. doi: 10.1016/j.cbpc.2005.05.006

O’Neill, E. C., Trick, M., Hill, L., Rejzek, M., Dusi, R. G., Hamilton, C. J., et al. (2015). The transcriptome of Euglena gracilis reveals unexpected metabolic capabilities for carbohydrate and natural product biochemistry. Mol. Biosyst. 11, 2808-2820. doi: 10.1039/c5mb00319a

Ono, K., Kawanaka, Y., Izumi, Y., Inui, H., Miyatake, K., Kitaoka, S., et al. (1995). Mitochondrial alcohol dehydrogenase from ethanol-grown Euglena gracilis. J. Biochem. 117, 1178-1182.

Osanai, T., Shirai, T., Iijima, H., Nakaya, Y., Okamoto, M., Kondo, A., et al. (2015). Genetic manipulation of a metabolic enzyme and a transcriptional regulator increasing succinate excretion from unicellular cyanobacterium. Front. Microbiol. 6:1064. doi: 10.3389/fmicb.2015.01064

Padermshoke, A., Ogawa, T., Nishio, K., Nakazawa, M., Nakamoto, M., Okazawa, A., et al. (2016). Critical involvement of environmental carbon dioxide fixation to drive wax ester fermentation in Euglena. PLoS ONE 11:e0162827. doi: 10.1371/journal.pone.0162827

Rodríguez-Zavala, J. S., Ortiz-Cruz, M. A., Mendoza-Hernández, G., and MorenoSánchez, R. (2010). Increased synthesis of $\alpha$-tocopherol, paramylon and tyrosine by Euglena gracilis under conditions of high biomass production. J. Appl. Microbiol. 109, 2160-2171. doi: 10.1111/j.1365-2672.2010.04848.x

Tan, Z., Zhu, X., Chen, J., Li, Q., and Zhang, X. (2013). Activating phosphoenolpyruvate carboxylase and phosphoenolpyruvate carboxykinase in combination for improvement of succinate production. Appl. Environ. Microbiol. 79, 4838-4844. doi: 10.1128/AEM.00826-13

Ueda, S., Kawamura, Y., Iijima, H., Nakajima, M., Shirai, T., Okamoto, M., et al. (2016). Anionic metabolite biosynthesis enhanced by potassium under dark, anaerobic conditions in cyanobacteria. Sci. Rep. 6:32354. doi: 10.1038/ srep32354

Werpy, T., and Petersen, G. (2004). Top Value Added Chemicals from Biomass Results of Screening for Potential Candidates from Sugars and Synthesis Gas, Vol. 1. Washington, D.C.: U.S. Department of Energy, 1-76.

Woodward, J., and Merrett, M. (1975). Induction potential for glyoxylate cycle enzymes during the cell cycle of Euglena gracilis. Eur. J. Biochem. 55, 555-559. doi: 10.1111/j.1432-1033.1975.tb02192.x

Yamada, K., Suzuki, H., Takeuchi, T., Kazama, Y., Mitra, S., Abe, T., et al. (2016). Efficient selective breeding of live oil-rich Euglena gracilis with fluorescenceactivated cell sorting. Sci. Rep. 6:26327. doi: 10.1038/srep26327

Yoshida, Y., Tomiyama, T., Maruta, T., Tomita, M., Ishikawa, T., and Arakawa, K. (2016). De novo assembly and comparative transcriptome analysis of Euglena gracilis in response to anaerobic conditions. BMC Genomics 17:182. doi: 10. 1186/s12864-016-2540-6

Conflict of Interest Statement: The authors declare that the research was conducted in the absence of any commercial or financial relationships that could be construed as a potential conflict of interest.

Copyright (C) 2016 Tomita, Yoshioka, Iijima, Nakashima, Iwata, Suzuki, Hasunuma, Kondo, Hirai and Osanai. This is an open-access article distributed under the terms of the Creative Commons Attribution License (CC BY). The use, distribution or reproduction in other forums is permitted, provided the original author(s) or licensor are credited and that the original publication in this journal is cited, in accordance with accepted academic practice. No use, distribution or reproduction is permitted which does not comply with these terms. 